

\section{THE NEW COLLECTIVE SPACES.}

Spaces of consum and leisure

Pedro BENTO

This article focuses on the impact produced by urban shopping centers and other architectural artifacts of leisure and consumption in its immediate environment. Unlike the current discourse and the media, the author defends the idea that sometimes these objects have a tremendously positive impact on the renewal of contemporary urban space, creating new buildings, improving public space and a substantial increase of existing economic activity.

Key words: Shopping centers, collective spaces, leisure and consumer artifacts 


\section{LOS NUEVOS LUGARES DE LA COLECTIVIDAD \\ Los espacios de consumo y ocio}

\section{Pedro BENTO}

El presente artículo está centrado en el impacto producido por los centros comerciales urbanos y otros artefactos arquitectónicos de ocio y consumo en su entorno próximo. Al contrario del discurso corriente y más mediatizado, se defiende la idea de que algunas veces estos objetos tienen un impacto tremendamente positivo en la renovación del espacio urbano contemporáneo, generando nueva edificación, mejoras en el espacio público y un incremento sustancial de la actividad económica existente.

Palabras clave: centros comerciales, espacios colectivos, artefactos de ocio y consumo 


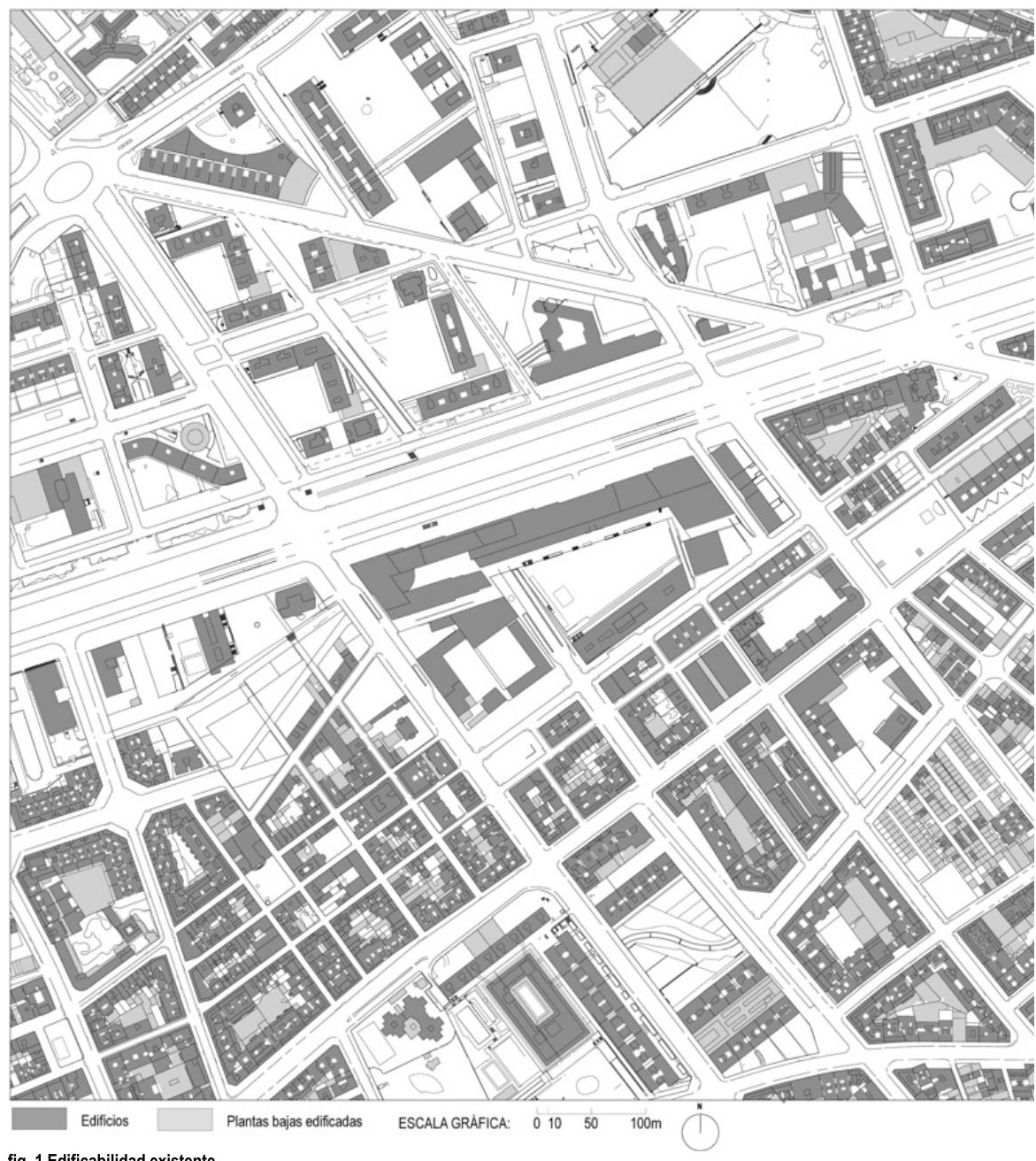

fig. 1 Edificabilidad existente 


\section{INTRODUCCIÓN}

El trabajo que se enseña de forma resumida en este artículo constituye una de las hipótesis más importantes de una investigación doctoral sobre espacios colectivos que se está llevando a cabo en el DUOT desde hace ya algunos años. En el referido trabajo doctoral, si las dos primeras hipótesis se centran fundamentalmente en la propia pieza arquitectónica y en las relaciones que esta establece con el entorno, la hipótesis que vamos a presentar seguidamente está enfocada exclusivamente en el espacio urbano sobre el cual el artefacto se implanta. Tras una presentación resumida del debate donde se esgrimen los argumentos a favor o en contra de estos equipamientos urbanos, se analiza un caso de estudio concreto - una área con $1 \mathrm{~km} 2$ alrededor del Centro Comercial l'Illa Diagonal - donde se observan en detalle cada uno de los criterios de análisis encontrados en el debate anterior, es decir: dinámica edificatoria, espacio público generado y actividad económica producida, para luego terminar con unas breves conclusiones.

\section{EL DEBATE SOBRE EL IMPACTO DE LOS CENTROS COMERCIALES URBANOS EN SU ENTORNO}

El debate sobre el impacto de los centros comerciales en su entorno urbano fue desde su inicio un debate muy intenso y polémico y con posiciones a menudo antagónicas. Frases como "they (shopping centers) suck the life of the shopping areas nearby", "shopping centers provide jobs and activity but they do so at the expense of virtually everithing else" y "they shut out the local community", etc. contrastan con frases como "they can be both regeneration schemes themselves and a catalyst for other non-retail regeneration projects", "new developments are creating a sense of place which is a natural extension of the city, not just a retail scheme, and through it, restoring and creating civic pride" y "it isn't just about building, it's about complete physical and social engagement with local communities". ${ }^{1}$

Para Benjamin R. Barber ${ }^{2}$, profesor en Walt Whitman Center for the Culture and Politics of Democracy en la Rutgers University, por ejemplo, los centros comerciales siguen siendo las plazas públicas privadas de las nuevas franjas de ciudad "privatopia" en donde la separación de la sociedad es usada para garantizar una seguridad plácida. Y que al entrar en un centro comercial cualquiera lo primero que se nos pide es que escondamos cualquier aspecto de nuestra identidad que no la del consumidor. 


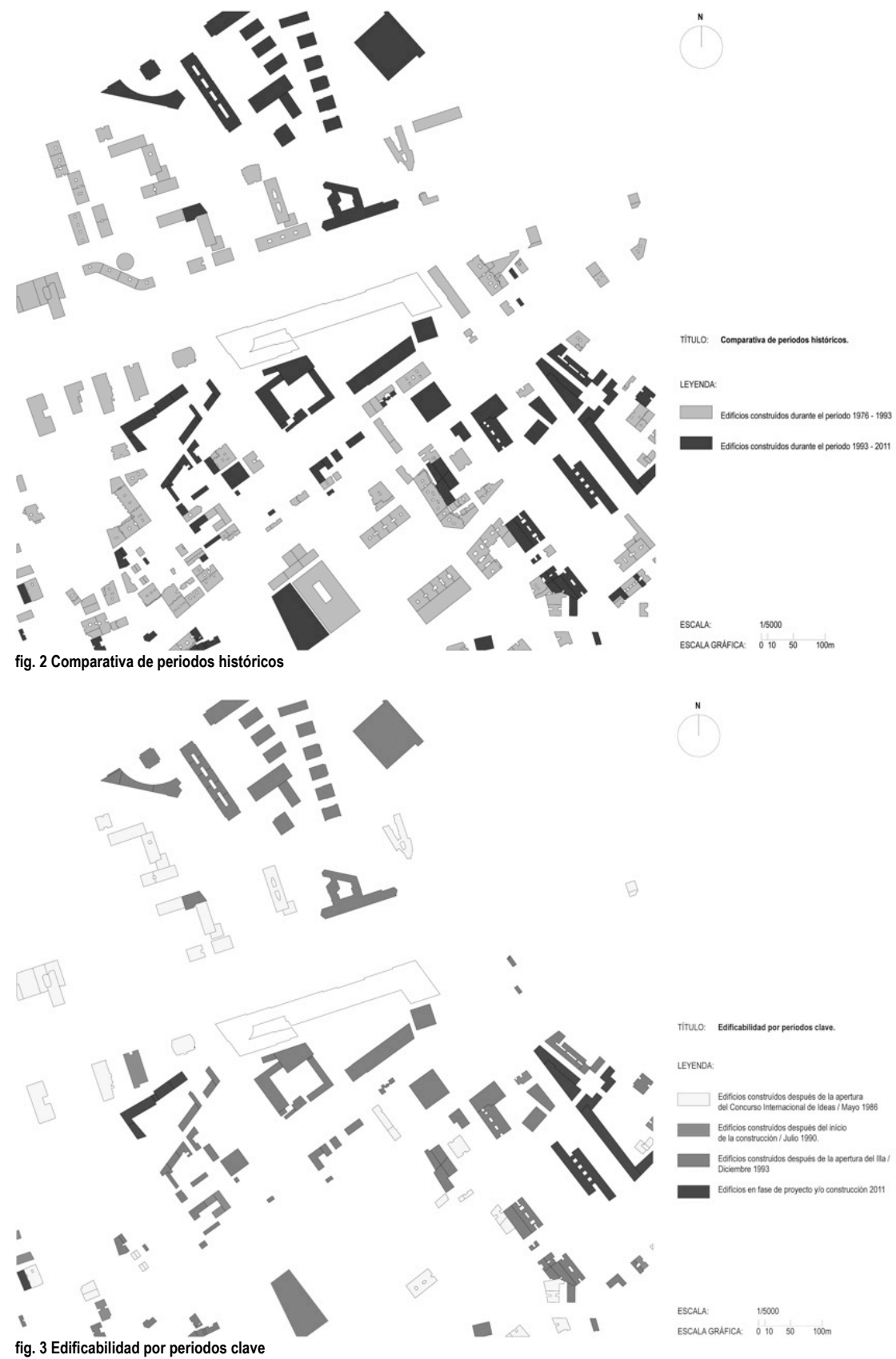


Por otro lado, la resistencia legal que han enfrentado las nuevas tipologías comerciales principalmente en los años 90 en Europa son la prueba suficientemente clara del aprecio o del sentimiento de amenaza que por estos nuevos edificios se tenía. ${ }^{3}$ La Ley de Comercio del 1996 en España requería un segundo permiso emitido por la autoridad gubernamental regional para construir nuevos centros comerciales; para citar algunos casos, en Bélgica era aprobada la Cadenas Law en 1994, para reducir el tamaño de estos equipamientos; en Dinamarca se aprobaban en 1997 el Plannig Act y las Environmental Impact Assessment Laws que obligaban los centros comerciales de grandes dimensiones a someter un informe sobre el impacto ambiental del proyecto en el momento de su tramitación; en Finlandia se aprobaba en 1998 la New Land Use and Building Act; en Francia se aprobaba en el 1973 la famosa Ley Royer que ponía una serie de límites a la proliferación de hipermercados y a tiendas con más de 1000m2, en 1990 la FISAC, en 1991/92, la Sapin Law (una adenda a la Ley Royer) que aunque no parase completamente el desarrollo de nuevos hipermercados lo ablandaba muchísimo. Luego en 1993 sale un decreto sobre los hipermercados, otro sobre las grandes tiendas de comida y una nueva adenda a la Ley Royer, la Ley Doublin elevando las restricciones a los supermercados, y por último en 1996 la Ley Rafferin que tenía como objetivo principal proteger el pequeño comercio.

Sin embargo, para Luis Felipe Alonso Teixidor ${ }^{4}$, Profesor de Urbanismo de la ETSA de Valladolid, refiriéndose acerca del conflicto con el pequeño comercio decía que "se ha planteado, no obstante con frecuencia, que los efectos de localización de estas piezas puedan inducir la destrucción de partes de tejido comercial de pequeña dimensión. Sin embargo, muchas de las evidencias no son del todo concluyentes en este sentido, al menos en lo que respecta a la intensidad de esos efectos negativos y su repercusión en el pequeño comercio tradicional'.

Por su parte, Enric Vives i Valls ${ }^{5}$ afirmaba que los centros comerciales funcionaban como dinamizadores del consumo y de su zona de ubicación "Si es cierto que un centro comercial de éxito activa el consumo, la consecuencia inmediata es la mejora de la actividad comercial en la zona de influencia en la cual este se ubica. Todo ello conlleva de modo inherente una mejora de la calidad de oferta, así como una mayor consolidación de la actividad comercial en las proximidades del centro".

Carles Carreras Verdaguer ${ }^{6}$, Catedrático de Geografia Humana del Departamento de Geografía Humana de la Universidad de Barcelona, afirmaba

\footnotetext{
${ }^{3}$ Ver el artículo de Chuihua Judy Chung y Juan Palop Casado, Resistance. En Project on the City 2. Harvard Design School Guide to Shopping. Taschen 2001. p.633-659.

4 "Actividad comercial y planeamiento urbanístico". En:Curso de Urbanismo de Areas Comerciales. Servicio de Publicaciones del Colegio Oficial de Madrid. Madrid 1989. p.53-74.

5 "La promoción comercial en la trama urbana consolidad". En:Curso de Urbanismo de Areas Comerciales. Servicio de Publicaciones del Colegio Oficial de Madrid. Madrid 1989. p.33-43.

6 "La funcionalitat de l'espai públic: nous espais de consum.". En: Espais públics. Mirades multidisciplinàries. Rosa Tello i Robira (coord.). Editorial Portic. Barcelona. 2002. p. 95-116.
} 
que efectivamente, los centros comerciales que se han instalado en Barcelona y en su área metropolitana tienen unas características que son relativamente originales. En primer lugar, una parte importante presenta una localización claramente urbana frente a la localización periurbana de los modelos de Francia o de Estados Unidos.

Son efectivamente solares céntricos de la ciudad que han permitido diversificar y aumentar la accesibilidad que incorpora normalmente el acceso a pie, conservando en algunos casos la trama urbana de la ciudad.

"D'altra banda, la localització céntrica ha ajudat en el manteniment de la vitalitat del centre comercial tradicional de la ciutat al llarg dels seus cinc quilómetres" y aduce que "la incorporació del disseny des de l'inici $i$ el treball encarregat a arquitectes que no bavien estat tècnics internacionals especialitzats de les grans distribuïdores han possibilitat la creació d'un model Barcelona de shopping center que podria entendre com una resistència local davant la pressió de la globalització".

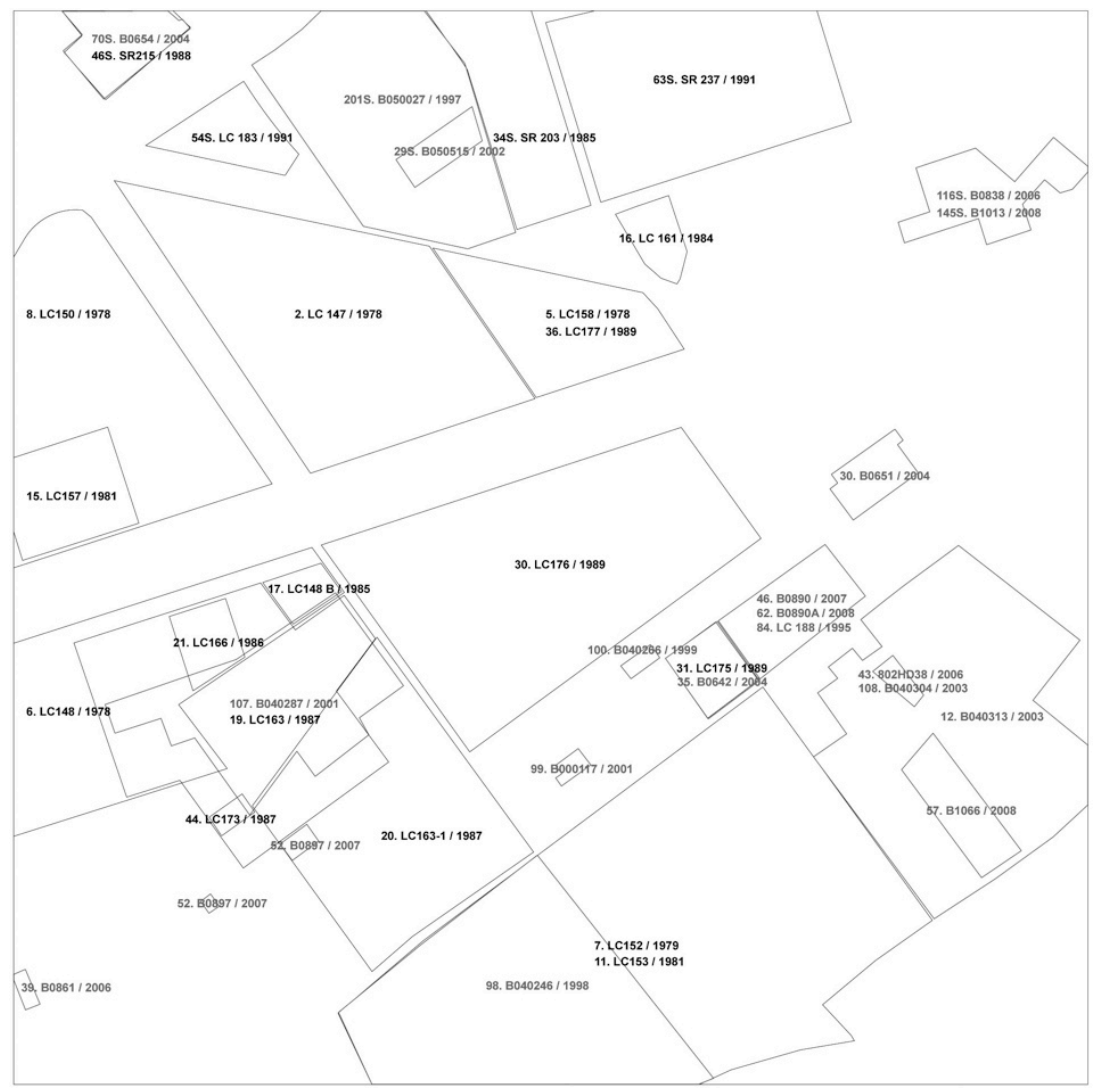

fig. 4 Espacio público y Urbanización. Modificaciones urbanísticas durante los periodos 1977-1993 y 1993-2011 
En definitiva, la idea de que los centros comerciales urbanos contribuyen siempre al empobrecimiento del centro de la ciudad y de la actividad económica local existente, no está del todo clara y nuestra hipótesis propone demostrar que, a menudo, es exactamente lo contrario: algunos centros comerciales y de ocio urbanos en la ciudad compacta pueden constituir verdaderos catalizadores $^{7}$ de urbanidad, y en algunos casos han llegado a ser los principales motores de importantes obras de renovación urbana llevadas a cabo en los últimos años. Sus efectos dinamizadores, al contrario de lo que acontece en la ciudad dispersa, a menudo han contribuido en la mejora del espacio público y en la dinamización de las actividades comerciales y terciarias en general del entorno, al margen de los efectos de los centros comerciales, en el pequeño y mediano comercio de una ciudad o población en su conjunto.

LEYENDA:

\section{Periodo 1977 - 1993}

2. LC 147 / 1978 Ordenación del poligono comprendido entre la Avenida Generalisimo Franco, Avenida Sarriá, Numancia y Agustina Saragossa. Adaptado al PGM.

5. LC158 / 1978 Estudio de nueva ordenación de la manzana limitada por la avenida Diagonal, Agustina Saragossa y avenida Sarrià.

6. LC148 / 1978 Estudio de Detalle del sector comprendido entre la Avenida del Generalisimo Franco y las calles de Numancia, Europa y Juan Guell.

7. LC152 / 1979 Delimitación de la unidad de actuación del sector comprendido entre las calles Ecuador, Taquigrafo Serra, Numancia Marques de Sentmenat y Travesera de Las Corts

8. LC150 / 1978 Estudio de Detalle de la manzana limitada por la avenida Generalísimo Franco, calle Sarasate, calle sin nombre, resultante de la
reparcelación de la supermanzana delimitado por la Gran Via de Carlosill, calle Numancia y plaza de la Reina Maria Cristina

11. LC153 / 1981 Estudio de detalle del poligono comprendido entre calle Ecuador, calle Taquigrafo Serra, calle Marqués de Sentmenat, calle Numancia y Travesera de Les Corts.

15. LC157 / 1981 PERI determinación de equipamiento sanitario del edificio existente en la Avenida Diagonal 656 a 660

16. LC 161 / 1984 Estudio de detalle de ordenación volumétrica del edificio $\mathrm{H}$ situado en la Avenida Dr. Fleming 4-6,

esquina a la calle Bori y Fontestá

17. LC148 B / 1985 Estudio de Detalle de la ordenación volumétrica del edificio a construir en el solar propiedad de INDUFISA S.A. con fachada a la avenida Diagonal 579-583 esquina a calle Numancia

19. LC163 / 1987 Pla especial d'ordenació volumètrica i millora del sector delimitat pel carrer de Numància, Avinguda Diagonal, carrer Gandesa, d'Europa y de Cabestany, entorn de la Plaça de la Concórdia limit inferior zona 14a i carrers Joan Gamper i Solà

20. LC163-1 / 1987 MPGM per ordenar l'ámbit definit pels carrers Numancia, Dr. Ibáñez, Anglesola i Vilamur. Acord del Conseller de Politica Territorial i Obres Públiques

21. LC166 / 1986 Estudio de Detalle de ordenación volumétrica de la finca 589-595 de la Avenida Diagonal

30. LC176 / 1989 Pla especial d'ordenació de I'llla Diagonal, definida pels carrers d'Entença, Déu i Mata, Numància i Avinguda Diagonal, formulat per "Inmobiliaria Diagonal Centro S.A."

31. LC175 / 1989 Plan especial Travesera de Les Corts 319-321 36. LC177 / 1989 ED d'ordenació de la parcel la de l'Avinguda Diagonal 640 44. LC173 / 1987 MPGM en el carrer Doctor Ibáñez núm. 27 LC180 34S. SR 203 / 1985 Plan Especial para la ordenación volumetrica de la unidad de actuación limitada por Rda. Gral. Mitre, c. Dr Flemming. Av. Sarria e instalaciones deportivas del RCD Español incorporando la zona de Equipamientos colindante prevista en el P.G.M.

46S. SR215 / 1988 Pla Especial d'Ordenació i Ampliacio de l'area

d'Equipament ocupada per la Clinica Corachan S.A. i Promotora Corachan S.A.

54S. LC 183 / 1991 Estudi de detall per a l'establiment d'alineacions i

rasants al carrer Casafranca, a Vallvidrera entre les cruilles amb el carrer

Can Basseda i la carretera de la Església.

63S. SR 237 / 1991 Pla Especial per al Desenvolupament d'un Parc Urba al Sector Piscines i Esports promogut per "Sotaverd S.A.".

\section{Periodo 1993 - 2011}

12. B040313 / 2003 MPGM en l'àmbit del sector de la Colònia Castells.

30. B0651 / 2004 MPGM per a r'Ordenació de L'llla delimitada pels carrers d'Entença, Déu i Mata, Taquigraf Garriga i Ptge. Cinc Torres.

35. B0642 / 2004 Pla especial urbanistic d'ordenacio d requipament de rilla Travessera de les Corts Bergada Taquigraf Garriga Prat de Rull

39. B0861 / 2006 PMU per a l'ordenacio volumetrica de la parcel.la situada al carrer de Masferrer, nums. 2-4, i al carrer de Joaquim Molins, num. 14.

43. 802 HD38 / 2006 MPGM creacio del sistema habitatge dotacional i concrecio dels sols als quals s'assigna aquesta qualificacio.

46. B0890 / 2007 PEU per a l'ordenació del recinte del Col.legi Pare Manyanet. ubicat a lilla delimitada pels carrers d'Entença, de la Travessera de les Corts. de Prat d'En Rull i de Taquigraf Garriga.

52. B0897 / 2007 MPE de protecció del patrimoni arquitectónic de la Ciutat de Barcelona al $\mathrm{Dt}^{\circ}$ de les Corts en els ambits del carrer de mejia lequerica $0^{\circ} 35-55$, Barcelona de la Concórdia $n^{\circ} 4-5$ i carrer del Remei $n^{\circ} 5$.

57. B1066 / 2008 PMU de la unitat de projecte UP-E de la MPGM en el SRI de la Colónia Castells.

62. B0890A / 2008 MPEU per a l'ordenació del recinte del Col.legi Pare Manyanet, ubicat a rilla delimitada pels carrers d'Entença, de la Travessera de les Corts, de Prat d'En Rull i de Taquigraf Garriga.

84. LC 188 / 1995 ED de compensació de volums de Pilla delimitada Travessera Les Corts, Prat d'En Rull, Taquigraf Garriga i Entença.

98. B040246 / 1998 ED de concreció de la cota de referència en planta baixa a la zona esportiva de lilila delimitada pels carrers de Vallsepir, Travessera de Les Corts, Numáncia i Marqués de Sentmenat.

99. B000117 / 2001 MPGM per a la Protecció del Patrimoni Arquitectónic de la ciutat de Barcelona

100. B040266 / 1999 Pla Especial de Compensacio de Volums de les Parcelles situades al carrer de Taquigraf Garriga, 87 - 89 .

107. B040287 / 2001 MPGM al sector de lentorn del carrer Anglesola

108. B040304 / 2003 Pla Especial d'Ordenació de I'Equipament situat entre els carrers d'Entença 275 - 293 i Montnegre 39

29S. B050515 / 2002 Pla Especial Urbanistic per a la concreció del tipus i les condicions d'edificació de l'Equipament situat al carrer de Can Rábia $n^{\circ} 7$ - 9

70S. B0654 / 2004 Pla especial urbanistica de l'àrea d'equipament ocupada per la Clinica Corachan carrer Buiggas $n^{\circ}$ 17-19 i Plaça de Manuel Corachan $n^{\circ} 4$

116S. B0838 / 2006 PEU d'ordenacio del subsol a la plaça de Wagner. 145S. B1013 / 2008 MPEU d'ordenació del subsöl de la Plaça Wagner. 201S. B050027 / 1997 MPGM en els terrenys del RCD Espanyol a I'Av. de Sarrià, de l'antic Camp de Tir i maniobres a Montcada i Reixach i del sector Verneda - Can Picas a Sant Adrià del Besós.

7 Utilizamos aquí la expresión Catalizador porque era la expresión utilizada por Víctor Gruen en aquellos principios de los años 50 en Estados Unidos y que expresa todavía hoy con gran fuerza el impacto que tienen estas piezas en la ciudad. 


\section{ANÁLISIS DE UN CASO DE ESTUDIO: L'ILLA DIAGONAL}

De antemano, cabe decir que seguramente la convocatoria de un concurso entre los principales estudios de arquitectura a nivel mundial y su difusión pública y debate cultural, en el caso de estudio de l'Illa Diagonal, son indicadores que favorecieron a una implícita mejor recepción del proyecto por parte de la sociedad en general y de la asociación vecinal del barrio de Les Corts en particular, desde su inicio.

\section{Dinámica Edificatoria}

El análisis de la dinámica edificatoria (realizada con base en la fecha de construcción de los edificios existentes, información disponible en la Cartografía Catastral de la Sede Electrónica del Catastro), nos permite constatar que desde la apertura del Centro Comercial l'Illa en diciembre del 1993 hasta mediados del 2011 se construyeron numerosos edificios. Aunque en el sector, debido al prestigio de la zona, perfil social de sus habitantes, precios del suelo y vivienda, y también escasez de suelo en Barcelona, de por sí también era de prever una cierta dinámica edificatoria. Curiosamente, aunque el número de edificios generados en el período homólogo anterior (1976-1993) sea más elevado, sospechamos que el Área Bruta de Construcción será bastante equivalente en los dos periodos (Fig. 1 y 2).

Por un lado, en el periodo 1976-1993 en la zona Norte de la Av. Diagonal se edificó masivamente, sobretodo el área entre esta última avenida, la Av. de Sarriá y Ronda General Mitre. Y, en el periodo posterior, se ocupó un área de tamaño similar entre la Av. Sarriá, la calle del Doctor Flemming y la misma Ronda del General Mitre, donde destacamos la ampliación del Equipamiento Deportivo DiR Piscinas i Deportes, el edificio compacto ocupado actualmente por Caja Madrid y que completa la fachada Norte de este tramo de la Diagonal, el nuevo equipamiento escolar Colegio Mayor de Sant Jordi y un conjunto importante de edificios de vivienda y oficinas en el lugar que antes ocupaba el Estadio del R.C.D. Español.

Por otro lado, la zona Sur de la Diagonal también sufrió una importante densificación con un carácter más disperso, tanto en el periodo 1976-1993 como en el 1993-2011. Para describir sumariamente esta transformación podríamos referirnos concretamente a tres grandes áreas: la Colonia Castells a levante; el núcleo histórico de Les Corts/ Av. Europa a poniente y la zona envolvente al equipamiento comercial l'Illa al centro.

Por otro lado, si analizamos los edificios construidos desde la fecha de apertura del concurso internacional de ideas de l'Illa en mayo de 1986, pasando por aquellos construidos desde el inicio de su construcción en julio de 1990, e incluyendo los generados después de la apertura del equipamiento al público y obviamente aquellos en fase de construcción, podemos quedar bastante sorprendidos (Fig. 3).

La observación de esta ilustración nos permite, entre otras cosas, admitir 
que tanto la construcción del Hospital de Barcelona como algunos de aquellos edificios plurifamiliares que van apareciendo en la zona Norte de la Diagonal, descritos anteriormente, como son las torres que van apareciendo en la Diagonal, entre el Corte Inglés y l'Illa y otros edificios localizados de forma dispersa a Sur del área de estudio se producen animados por el desarrollo económico e inmobiliario que se vive en el momento. Pero también por las buenas señales que suscita la apertura de un concurso de ideas de l'Illa y la posterior construcción del centro comercial.

Parece así bastante evidente que en este caso la aparición del centro comercial no vino a empobrecer / debilitar la estructura edificada del entorno o retirar valor al espacio urbano sino todo lo contrario. Toda el área se encuentra reestimulada y confiada en el suceso urbanístico de las diversas operaciones inmobiliarias.

\section{URBANIZACIÓN Y ESPACIO PÚBLICO}

En un primer periodo, de 1976-1993, las aprobaciones definitivas de los instrumentos de planeamiento se concretan sobre todo en Estudios de Detalle y algunos Planes Especiales localizados de forma más o menos central con relación al área de estudio, entre los cuales destaca el área de intervención de l'Illa y su envolvente inmediata (30. LC176/1989 Pla especial d'Ordenació de l'Illa Diagonal formulado por la "Inmobiliária Diagonal Centro S.A."). En el período siguiente 1993-2011 los instrumentos de planeamiento son sobretodo Modificaciones del Plan General Metropolitano y Planes Especiales localizados de forma más periférica respecto al artefacto, a saber en la zona de la Colonia Castells, en la zona del antiguo Estadio del Español, Av. Europa/ Anglesola y algunos espacios públicos dispersos por todo el área de estudio, como el de la Plaza Can Rosés o la recién inaugurada Plaza Wagner (Ver ilustración 4).

Sin embargo, si vamos más atrás y buscamos todas las aprobaciones definitivas de los diferentes Instrumentos de Planeamiento existentes desde la fecha de apertura del concurso internacional de ideas en mayo de 1986 hasta la actualidad veremos que el número de modificaciones urbanísticas ha aumentado en 30, lo que representa el $150 \%$ respeto el número de modificaciones anteriores (Ver ilustración 5).

Por lo tanto, lo que estos dibujos tratan de explicar es que la implantación del Equipamiento Comercial no supuso una amenaza a la urbanización del entorno ni a su consolidación, sino todo lo contrario. Además se puede verificar es que las acciones urbanizadoras se han producido de forma natural, con alguna desaceleración, como es normal, dado que ya se había urbanizado una parte importante del área de intervención y que esta ha permitido reforzar y continuar el proyecto urbano de la ciudad.

Otra forma de poder evaluar el impacto urbanístico de un determinado centro comercial y de ocio en la cualificación de la ciudad y de su espacio público puede ser precisamente la de identificar los nuevos espacios públicos generados exnovo o por acciones de reforma o recalificación durante los 
diferentes periodos, anterior o posterior a la apertura del equipamiento.

Este análisis en particular evidencia de una manera sorprendente la hipótesis formulada al explicar que los espacios públicos producidos de nuevo, o aquellos otros mejorados por diversas acciones de reforma son en mayor número y de mayor importancia y significación urbana durante el periodo de apertura del equipamiento hasta la actualidad (1993-2011) que los espacios públicos generados en el periodo anterior. (Ver ilustración 6)

TITULO: Espacio público por períodos clave. LEYENDA:

\section{Espacio Público generado durante el periodo 1976 - 1993}

1. Definición y Ejecución de las calles Marti i Juliá, de Flos i Calcat y de Josep Irla i Bosch. Años 70.

2. Definición y Ejecución de las calles Caravel.la la Niña, Nau Santa Maria, de la Caravel.la la Pinta. Años 70.

3. Jardins de Joaquim Ruyra. Parcs i Jardins. Años 70.

4. Jardins de Can Batiori . 1987

5. Jardins de Blanca Selva i Henry. 1987

6. Jardines de Catalana Occident. 1981

7. Jardins de Piscinas y Deportes

8. Jardins de Clara Campoamor

9. Jardins de Ferran Soldevilla

10. Plaça del Hotel Hilton. Viaplana. 1990

11. Plaça de Valdivia . 1990

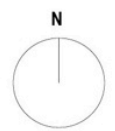

ESCALA GRÁFICA: $\quad \begin{array}{llll}010 & 50 \quad 100 \mathrm{~m}\end{array}$
Espacio Público generado con el equipamiento en Diciembre del 1993

12. Extensión cl Constança.

Manuel de Solá y Rafael Moneo. Dic. 1993.

13. Mejora de las $c /$ Diagonal, Numancia, Deu i Mata y Pau Romeva. Manuel de Solá y Rafael Moneo. Dic. 1993.

14. Jardins de Sant Joan de Déu.

Manuel de Solá y Rafael Moneo. Dic. 1993.

Espacio Público generado despues de Diciembre de 1993

15. Cualificación del nucleo histórico de Les Corts a través de la peatonalización de las calles del Remei, de Solà, de Deu i

Mata, de Taquigraf Garriga, de Cabestany, del Doctor Ibañez, de Vilamur y de Joan Gamper y de la Plaza de la Concordia.

Districte de Les Corts.

16. Proyecto de Mejora Urbana de las calles de Nicaragua, Taquigraf Marti, del Berguedá, del Prat d'en Rull, del Marques de Sentmenat, de Taquigraf Garriga, de la Travessera de Les Corts (des de I'Av. de Sarriá fil al cl d'Entença), Montnegre, de l'Ecuador. Districte de Les Corts. Entre 2008 y 2011.

17. Recualificación de la Av. Numancia entre la Plaza del Prat de la Riba y la Av. Diagonal.

18. Proyecto de Mejora de la Ronda de Dalt.

19. Creación del Pasaje de Ricard Zamora.

20. Urbanització de la plaça Rosés. Dist. de Les Corts. Sep. 2010

21. Reforma de la Plaza del Doctor Ignasi Barraquer. Dist. de Les Corts

22. Urbanització de la Plaça de Caba.

23. Urbanització de las Plazas de Wagner y Joan Llongueras 201

24. Creacion de la Plaza d'Ángel Rodriguez.

25. Urbanización de la Plaza de Ricard Zamora.

26. Creacion de la Plaza de Belianes.

27. Reforma de la Plaza de Prat de la Riba

28. Reforma de la Plazas Comas y peatonalización de las calles Taquigraf Marti y Mansferrer.

29. Jardins de Gaietà Renom. Bagursa. Oct. 2008

30. Jardins de Can Cuiás.

31. Jardins de Les Infantes.

32. Jardins de $\mathbf{M}^{\mathrm{a}}$ Dolors Bargalló i Serra.

33. Jardins d'Olga Sacharoff. Casamor y Gabas. 1994

34. Jardins de Joan Vinyol. Casamor y Gabas. 1994

35. Jardins del Camp de Sarrià

36. Jardins del Doctor Castelló

37. Jardins del Doctor Samuel Hahnemann.

38. Parque de Les Corts. Carme Fiol.
Espacio Público en fase de proyecto o execución

39. Prolongamiento de la c/ Europa y Doctor Ibañez, Urbanización de la cl d'Anglesola y Recuperación de la c/ Can Segalar en el ámbito del Plan Europa-Anglesola.
Bagursa. 2011-2012

40. Proyecto de Mejora Urbana de las calles Entença, Montnegre y de Morales y de la Plaza del Carme en el ámbito del Plan Colónia Castells

Districte de Les Corts. 2011 y 2012

41. Plaza prevista en el Plan Colónia Castells. Bagursa. 2012 42. Jardins previstos en el Plan Europa-Anglesola. Bagursa. 2011-2012

fig. 6 Obras de Cualificación y Renovación de espacio público por periodos clave 


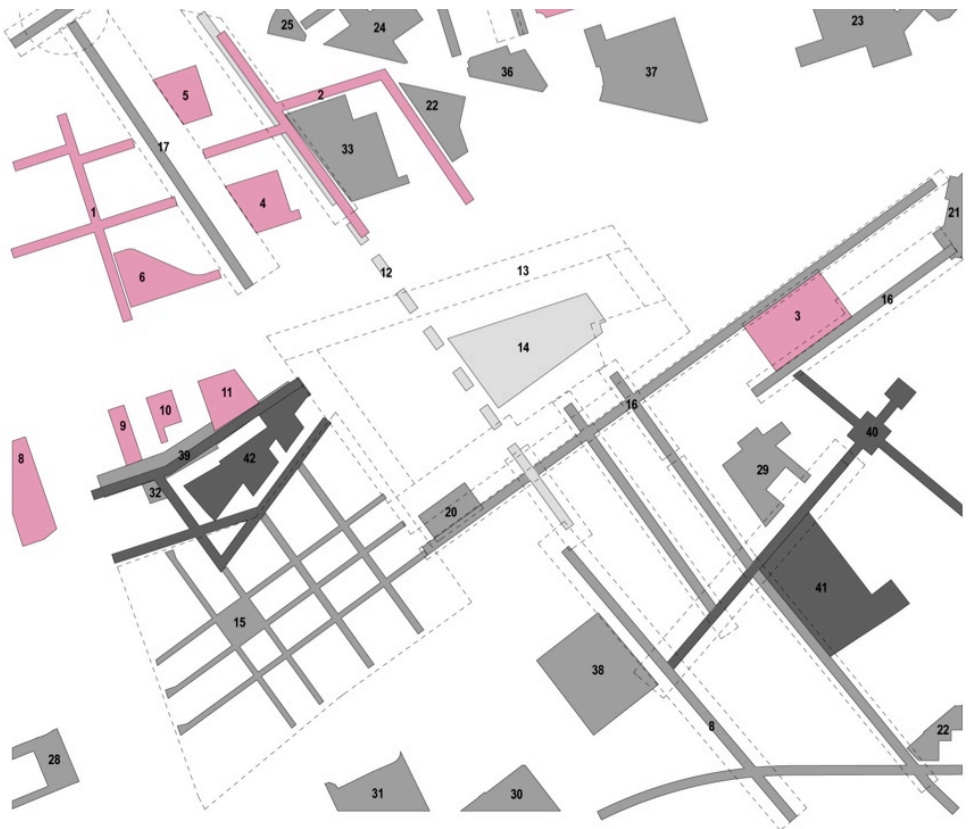
畞

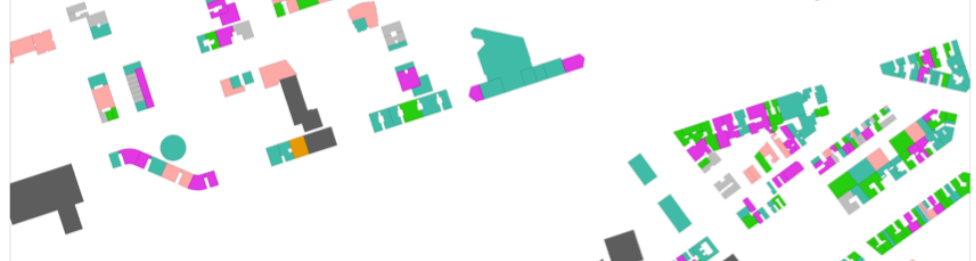
1

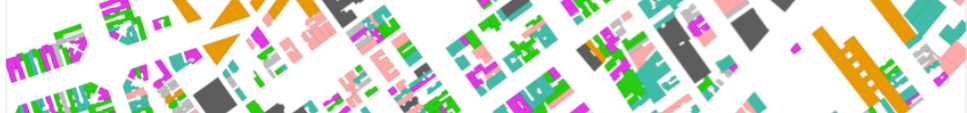
Atrie 1 a at Accividates de Restaracoion
Equipaimontos 


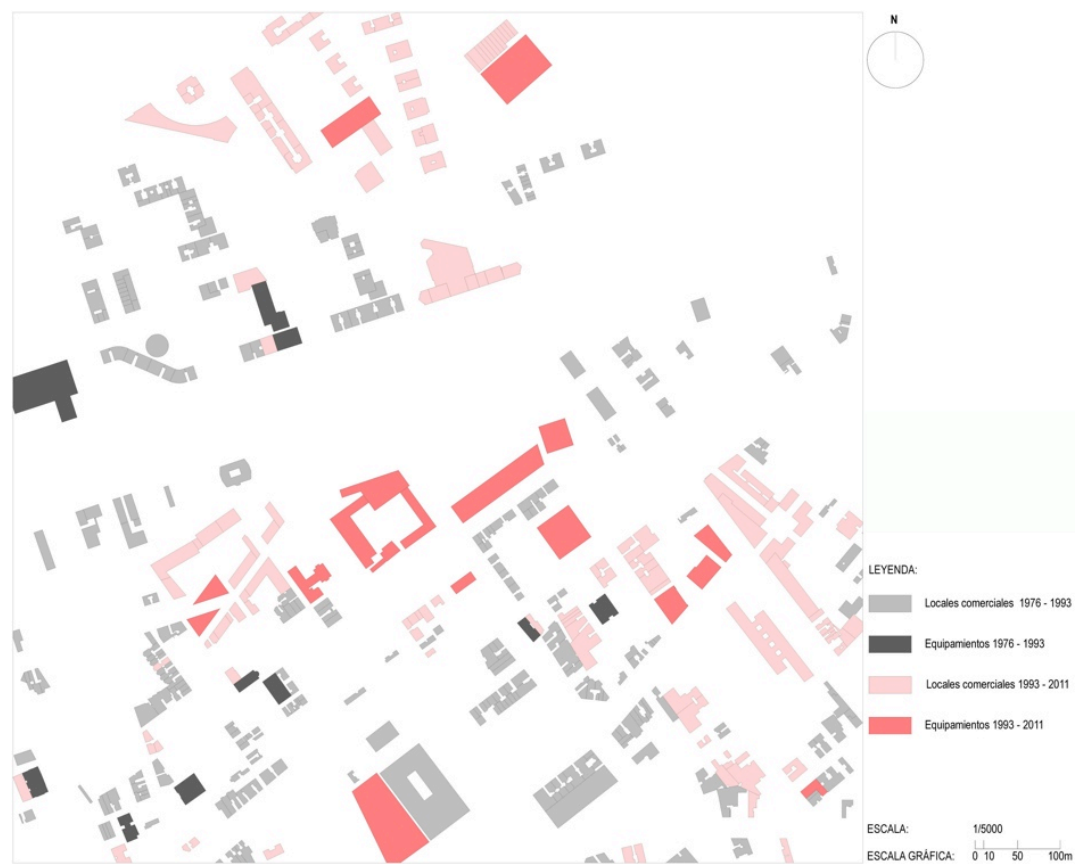

fig. 8 Locales y equipamientos nuevos generados por la nueva edificación construida durante los periodos 1976-1993

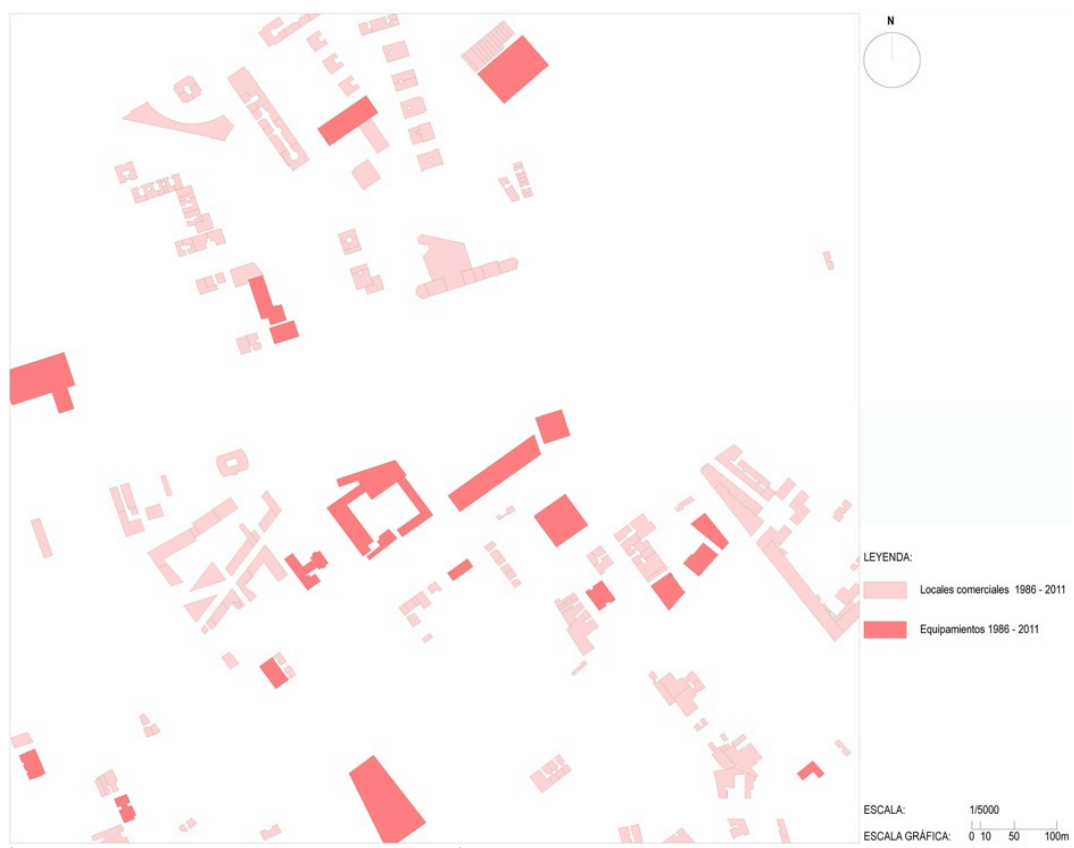
fig. 9 Locales y equipamientos nuevos generados por la nueva edificación construida desde la apertura del concurso en mayo de 1986 
Es decir, mientras que en periodo 1976-1993 se produjeron apenas una docena de espacios públicos, localizados principalmente en aquellas zonas de nueva urbanización, en diciembre del 1993 se abren con el equipamiento importantísimas infraestructuras y espacios públicos urbanos nuevos. Así, después de diciembre de 1993 se producen por lo menos dos docenas de recalificaciones urbanas, todo tipo de mejoras, y reformas, ampliando de forma singular las acciones creadoras de espacio público en el área de estudio.

Lo que sí es bastante evidente en este análisis es que a pesar de saber que el proceso de mejora de los espacios públicos urbanos llevados a cabo por el ayuntamiento se debe a la puesta en práctica de una política municipal de espacio público, los efectos de esa misma política se acentúan fuertemente en el periodo post-artefacto.

En definitiva, pensamos que la implantación del importante espacio colectivo privado de l'Illa vino a potenciar de forma excepcional y completamente inusual el espacio colectivo público, aplicándose en este caso a la perfección el conocido dicho popular "la calidad conlleva calidad".

\section{Actividad Económica}

Tras el análisis riguroso de la actividad económica se confirma la presencia de un área bastante activa y dinámica de la ciudad. La gran diversidad de usos y la densidad de actividades económicas son sus principales características. De acuerdo con el levantamiento realizado in situ hemos detectado la presencia de un $17,1 \%$ de restauración, $28,1 \%$ de comercio, $27,9 \%$ de servicios, $12,0 \%$ de oficinas, $1,9 \%$ de otros locales y equipamientos, $9,2 \%$ de locales vacíos en estado de alquiler o venta y $3,2 \%$ de equipamientos en un total de 1254 locales y equipamientos (Ver ilustración 7).

Tanto la restauración, como el comercio o los servicios se encuentran distribuidos por el territorio de forma bastante homogénea, aunque exista una mayor densidad en la zona Sur de la Diagonal, debido a una correspondencia directa con el propio grano más pequeño de su tejido urbano. Las oficinas están concentradas a ambos lados de la Diagonal en uno y dos núcleos respectivamente. Las oficinas de mayor entidad ocupan sobretodo la zona Norte de la Diagonal mientras que las oficinas de menor dimensión ocupan la zona Sur. Los locales y equipamientos no incluidos en los usos anteriores, corresponden a dos áreas que están siendo objeto de reforma y actualmente se encuentran en fase de construcción. Los locales vacíos detectados en el área de estudio surgen a ambos lados de la Diagonal aunque con mayor incidencia en el lado Sur por la misma razón de los comercios, servicios y restauración. Una parte importante de los locales vacíos encontrados en la zona Norte de la Diagonal siguen vacíos porque se ve que acaban de ser edificados. Los equipamientos aparecen en mayor número en la zona Sur de la Diagonal pero los de mayor dimensión se localizan a Norte.

Sin embargo, mirando con un poco más de detalle el plano podemos distinguir dos zonas comerciales distintas respectivamente, al Norte y al Sur de la Av. Diagonal. En la zona Norte encontramos bastantes oficinas, servicios 
diversos y grandes equipamientos urbanos. Al Norte de la Ronda de Dalt y en las manzanas definidas por la Av. Diagonal, c/ Ganduxer, c/ Francesc Pérez Cabrero y Av. Pau Casals se registra una actividad comercial y una mezcla de usos intensa.

La zona donde se detectó menor intensidad de usos y por consiguiente menor actividad comercial corresponde a la manzana situada entre el Parque de Piscinas y Deportes y la Av. Diagonal, donde en el año 69 se construyeron unos edificios de vivienda de unas veinte plantas promovidas por la Caja de Pensiones, proyectadas por Javier Subías, Pedro López Iñigo y Guillermo Giráldez con una imagen bastante sugestionada por el periodo moderno.

El Parque de las Piscinas y Deportes se benefició de un espacio de gran actividad aunque al principio motivó una gran polémica y debate ciudadanos con el complejo de ocio recientemente inaugurado que en un mismo espacio integra piscinas, pistas de tenis, gimnasio, cines, bares y restaurantes con terrazas.

Los jardines del antiguo campo de futbol de Sarriá y la contigua plaza de Ángel Rodríguez, lugares donde antes se situaba el Estadio del Español, son espacios controlados diariamente debido a la instalación de un supermercado Caprabo de medianas dimensiones situado al final del nuevo conjunto residencial, lo que demuestra también que la apertura del Centro Comercial l'Illa no agotó el mercado alimentario existente. Todo lo contrario, el Mercado de Les Corts acaba de realizar una importante reforma, el Centro Comercial Pedralbes, con motivo de su 25 cumpleaños, ha hecho una renovación interior total y que sepamos el Corte Inglés localizado unos metros más arriba en la Av. Diagonal no perdió clientes.

Al Sur de la Av. Diagonal el tamaño del comercio tiene una correspondencia directa con el grano urbanístico existente. Se trata de una estructura comercial densa y muy diversa y desde nuestra perspectiva ha salido enriquecida con el mecanismo de atracción comercial que l'Illa generó y sigue generando.

El levantamiento de todos los locales del área de estudio permite ilustrar la idea que venimos defendiendo a lo largo de este trabajo: el gran artefacto comercial y de ocio no deterioró el comercio local sino que lo incrementó (Ver ilustraciones 8 y 9).

De hecho, como se puede verificar en los dibujos siguientes, el comercio local está bastante disperso por el territorio, con un total de 352 locales. Cabe decir que se encontraron algunos locales vacíos en puntos específicos del área de estudio - a saber, en la Colonia Castells y en la zona de la Av. Europa / c/ Anglesola - porque son dos áreas que están en proceso de sustitución edificatoria. Los servicios suman 350 locales y la restauración 215.

Mirando ahora el análisis que contrasta los locales y equipamientos nuevos generados por la nueva edificación en los dos periodos (Ver ilustración 9) resulta bastante evidente que los locales construidos en el periodo anterior son en mayor número que aquellos construidos en el periodo siguiente, sin embargo los grandes equipamientos son más numerosos en el periodo posterior a la apertura del artefacto que en el periodo anterior. 
Esta situación no resulta extraña porque efectivamente la mayor parte de los edificios nuevos habían sido construidos en el periodo 1976-1993. Pero si miramos el plano siguiente, que ponen de manifiesto todos los locales construidos desde la apertura del concurso en mayo de 1986, vemos que estos aumentan.

Es decir, aunque se registre un mayor número de locales nuevos en el primer periodo 1976-93 vemos que una parte significativa de esos locales se producen con el anuncio de apertura y construcción del equipamiento entre el año 1986 y el año 1993.

Concluimos, por las razones expuestas, que en este caso, el Centro Comercial no dañó la estructura comercial existente sino que la consolidó directa e indirectamente como se puede verificar en los análisis gráficos anexos.

\section{CONCLUSIONES}

En definitiva, cabe dejar constancia de que los espacios colectivos en la ciudad compacta, y en particular los centros comerciales y de ocio, son unos espacios complementarios de los espacios públicos que interesa potenciar. Que si estos artefactos son proyectados con determinadas atenciones urbanísticas, seguramente van a crear sinergias con los espacios públicos tradicionales, ampliando significativamente la esfera pública y aumentando la actividad colectiva. Y por último, que algunos centros comerciales y de ocio tienen un impacto tremendamente positivo en la consolidación del entorno y en el fortalecimiento de la compactación urbana, como es el caso de l'Illa. Razón por la cual, los poderes públicos deberían perder las infundadas supersticiones a la incorporación de los mismos dentro de la ciudad compacta y quizá tener una participación más activa en su promoción y gestión. Esto sí, bajo un control, tanto en su diseño propio como en el de su sintaxis urbana. Como epílogo me gustaría añadir que quizá aquella ley fundamental de las polaridades que estructura cualquier centro comercial (dos polaridades que generan un flujo) también se pueda aplicar en la ciudad. En este caso los polos podrían ser los propios artefactos comerciales y de esta forma se podrían regenerar importantes fragmentos urbanos. 


\section{Referencias bibliográficas}

AA.WV. Curso de Urbanismo de Áreas Comerciales. Madrid: Servicio de Publicaciones del Colegio Oficial de Madrid. 1989.

AA.VV. Espais Públics. Mirades Multidisciplinaries. Rosa Tello I Robira (coord.). Editorial Portic. Barcelona. 2002.

AA.VV. Project on the City 2. Harvard Design School Guide to Shopping. Edición a cargo de Chuihua Judy Chung y Sze Tsung Leong. Koln: Taschen, 2001.

BENTO, Pedro. Los nuevos lugares de la colectividad. (Propuesta de Tesis Doctoral). Barcelona: DUiOT. 2011

CRAWFORD, Margaret. Blurring the boundaries: public spaces and private life, en J. Chase, M. Crawford and J. Kaliski (ed.) Everyday Urbanism. New York: Monacelli Press, 1999.

CRAWFORD, Margaret. "The World in a Shopping - Mall." In Variations on a Theme Park: The New American City and the End of Public Space. Edited by Michael Sorkin, 3-30. New York: Hill and Wang, 1992.

DEAN, Ptolemy y DOBSON, Rob. "Can Shopping Centres be used to regenerate cities?", En bdonline.co.uk. 7 Nov 2008.

GRUEN, Victor. Centers for the Urban Environment: Survival of the Cities. N. York: Van Nostrand Reinhold, 1973.

GRUEN, Victor, and Larry Smith. Shopping Towns USA: The Planning of Shopping Centers. N. Y.: Reinhold, 1960.

GRUEN, V., and L. P. Smith. "Shopping Centers: The New Building Type." Progressive Architecture 33 (June 1952): 66-94.

OLIVEIRA, Margarida. www.ciudad.consumo. El impacto de las redes de consumo en la reorganización del espacio urbano contemporáneo del Area Metropolitano de Lisboa, Lisboa, 2005

SMILEY, David J., ed. Sprawl and Public Space: Redressing the Mall. Washington: NEA, 2002.

SMITH, P. J. "Coping with Mega-Mall Development: An Urban Planning Perspective on West Edmonton Mall." Canadian Geographer 35 (1991): 295-305.

SOLÀ-MORALES, Manuel de. Espacios públicos y espacios colectivos. Un nuevo reto: urbanizar lo privado. En La Vanguardia, 12 de maig de 1992, Suplemento Cultura y Arte, pg. 4-5

TOBELLA FARRAN, Lluís. (Tesis Doctoral) L'Illa Diagonal de Barcelona. Análisi de questions del projecte. Barcelona, 2002.

WALL, Alex. Victor Gruen. From Urban Shop to New Clty. Barcelona: Actar, 2005. 